Saudi Journal of Medical and Pharmaceutical Sciences

Abbreviated Key Title: Saudi J Med Pharm Sci ISSN 2413-4929 (Print) |ISSN 2413-4910 (Online) Scholars Middle East Publishers, Dubai, United Arab Emirates Journal homepage: https://saudijournals.com/sjmps

Case Report

\title{
A Seldom Tuberculosis Localization: About 1 Case
}

Najoua Belhaj $^{1 *}$, Razika Bencheikh², Ikram Boumendil ${ }^{1}$, Mohammed Anass Benbouzid ${ }^{2}$, Abedelilah Oujilal ${ }^{2}$, Leila Essakalli $^{2}$

${ }^{1}$ Resident Physician in otorhinolaryngology, Department of Otorhinolaryngology, Head and Neck Surgery, Ibn Sina University Hospital, Faculty of Medicine, Mohammed V University, Rabat, Morocco

${ }^{2}$ Professor of Otorhinolaryngology, Department of Otorhinolaryngology, Head and Neck Surgery, Ibn Sina University Hospital, Faculty of Medicine, Mohammed V University, Rabat, Morocco

DOI: $10.36348 /$ sjmps.2020.v06i04.008

| Received: 16.03.2020 | Accepted: 23.03.2020 | Published: 25.04.2020

*Corresponding author: Belhaj Najoua

\section{Abstract}

Primary nasopharyngeal tuberculosis is rare, we present an observation revealed by a pseudo-tumor aspect posing the problem of differential diagnosis with malignant lesions of the cavum.

Keywords: Seldom Tuberculosis Localization.

Copyright @ 2020: This is an open-access article distributed under the terms of the Creative Commons Attribution license which permits unrestricted use, distribution, and reproduction in any medium for non-commercial use (NonCommercial, or CC-BY-NC) provided the original author and source are credited.

\section{INTRODUCTION}

Tuberculosis in MOROCCO represents a public health problem, its extra pulmonary localizations are unusual and misleadingly difficult to diagnose. It is one of the most widespread infectious diseases in the world with approximately 9 million new cases per year, causing 2 million deaths.

Its incidence has increased since the development of the AIDS epidemic, the increase of demography, poverty and migration of populations. Tuberculosis is a specific infectious disease caused by a mycobacterium: the Koch bacillus. It is a condition that still very frequent in our country in an endemic state, the pulmonary form is by far the most frequent; extra pulmonary forms represent $15 \%$; its incidence oto laryngological localizations is a relatively rare, and if the cervical lymph node form is the most encountered, other more rare localizations can be seen.

\section{Case Report}

A young patient, 20-year-old, consulted for a nasal obstruction evolving in a context of apyrexia and general condition preservation with episodes of epistaxis of low abundance .

Clinical examination showed a decreased nasal flow on both sides with an inflamed nasal mucosa

On cervical palpation there was no lymphadenopathy. The nasofibroscopia showed a bulging process of the cavum posterior wall.

A biopsy was made; the histological result was in favor of an epithelio-giganto-cellular granuloma with caseous necrosis, without malignancy signs. Biological check-up showed moderate inflammatory syndrome (CRP \& SR were slightly increased), tuberculin IDR was positive and chest X-ray was normal.

The scanner showed a thickening of the cavum posterior wall moderately enhanced after contrast product injections stretched over a height of $2.6 \mathrm{~cm}$ : in front it comes into contact with the choanes and the lower tip of the vomer, laterally it respects the tubular orifices and Rosenmüller's dimples and parapharyngeal spaces, and behind it respects the para-vertebral space. 


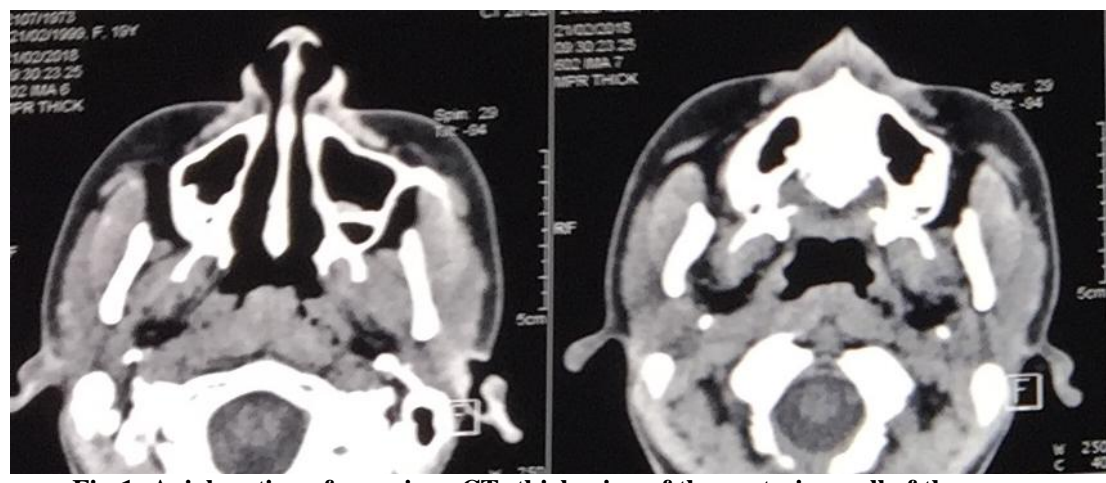

Fig-1: Axial section of nasosinus CT: thickening of the posterior wall of the cavum

\section{DiscuSSION}

The nasopharyngeal Tuberculosis refers to all of the progressive granulomatous type lesions of the mucosa following infection with the bacillus of koch [1].

It is a rare localization of the disease in its primitive form although the cavum is richly vascularized. The first case described was by GRAFF in 1936 [1].

The inoculation of the ENT site by the Koch bacillus can be done by several ways:

- The hematogenous pathway from a contaminated site, especially the pulmonary.

- The lymphatic route from a loco regional mucosal lesion

- The duct via the bronchial sputum containing the bacilli; It's upward diffusion.

- The direct route from the external environment, this mechanism explains the rare primitive forms.

These different inoculation mechanisms explain the lesional topographic diversity and the possibility of forms with multiple locations [2].

Tuberculosis with nasopharyngeal localization mainly affects young people; clinically, the symptomatology is dominated by nasal obstruction, epistaxis and rhinorrhea. Otological signs can be seen such as unilateral serous mucosal otitis. Sometimes associated cervical lymphadenopathy, they are most often bilateral under digastrics $[1,3,4]$.

The most frequent endoscopic aspect is that of a budding or ulcer formation, essentially posterior superior. CT is non-specific and can point a malignant process of the nasopharynx, hence the importance of performing systematic cavum biopsies.

Originally described by Giovanni Morgagni in 1761 on autopsy data; however nasosinus tuberculosis remains a rare entity estimated at $1 \%$ of tuberculosis of the head and neck [2].
Nasal tuberculosis can be classified in spontaneous nasal tuberculosis following pulmonary tuberculosis and primary nasal tuberculosis which occurs without prior pulmonary involvement. This last entity remains rare given the bactericidal action of the nasal secretions and the protection offered by ciliary movements [2].

The most frequent symptoms include cervical lymphadenopathy, weight loss, fever, epistaxis, nasal obstruction, hearing loss, otalgia, tinnitus, postnasal drip, and night sweats nasal obstruction, rhinorrhea, epistaxis as noted in our patients as well as the formation of crusts. The most interested sites are the cartilaginous portion of the nasal septum and the choanaes $[3,4]$.

The lesions can take the form of a granulomatous proliferative tissue or an ulceration which can lead to confusion with Wegener's granulomatosis or Sarcoidosis; this explains the importance of the anatomopathological and bacteriological examination in order to confirm the diagnosis [5].

The positive diagnosis of cavum tuberculosis can only be confirmed by histological examination with the presence of epithelio-giganto-cellular granulomas with caseous necrosis.

All the endoscopic, clinical and radiological clinical signs are especially in favor of a tumor etiology, need to perform several biopsies in different places to be able to eliminate a nasopharyngeal carcinoma or a combination that remains possible but exceptional.The germ's identification by direct examination or after culture on a specific medium requires 4 to 6 weeks and can prove to be unsuccessful so the biopsy fragment is systematically cultured on Lowstein medium in order to isolate the germ, type the species and carry out a very useful antibiogram in resistant forms or in the event of a history of antituberculosis treatment or in a particular field (HIV). Other biological processes currently exist; faster than conventional culture: The search for tuberculo-stearic 
acid by chromatographic method and PCR (polymerase chain reaction) gene amplification makes it possible to detect even faster in less than 12 hours and can sometimes decide in front of an epithelio-giganticcellular granuloma without caseous necrosis[1, 2, 6].

Gene expert is the only way to answer two questions is it or not tuberculosis and if it is, it detects eventually resistance to rifampicin.

Computed tomography (CT) images show a large mass, a lobulated mass, or irregular soft tissue thickening of the nasopharynx. Magnetic resonance imaging (MRI) is useful for soft granulomatous formation. On MRI, nasopharyngeal tuberculosis is seen as either a polypoid mass of the adenoids or diffuse thickening of the mucosal wall of the nasopharynx [4].

Once diagnosis of pharyngeal $\mathrm{TB}$ is confirmed, search for other locations is essential to adjust the treatment. Seeking HIV should be systematic [9].

The primary cavum tuberculosis treatment is medical based on anti bacillary poly chemotherapy, the classic combination used is a quadritherapy based on rifampicin, isoniazid and pyrazinamide and ethambutol for 2 to 3 months and followed by a relay for 4 to 6 months with a dual therapy (isoniazid and rifampicin) [8].

Therapeutic efficacy is based on the regression of clinical and endoscopic signs. Any abnormal development must evoke resistance or a possibility of coexisting neoplastic affection; therefore it is necessary to do iterative biopsies. The risk of relapse is estimated at $1 \%$, mainly due to the appearance of multi-resistant BK strains $[6,2]$

The recommended prevention method to avoid tuberculosis in children is the BCG vaccine given at birth or as soon as possible after birth, before the age of 12 months [8].

Vaccination and pasteurization of milk reduced the incidence of pharyngeal TB until the mid1980s, since then, other factors, such as human immunodeficiency virus (HIV) and the development of resistant strains seem to work in the opposite direction [9].

\section{CONCLUSION}

The cavum tuberculosis is an uncommon condition. Its symptomatology is non-specific and its diagnosis is often made on the occasion of a pseudotumor lesion biopsy. Its treatment is medical. It must be well managed in order to minimize the selection of resistant strains. The best treatment remains preventive, based on BCG vaccination and early detection of tuberculosis of the ENT sphere.

\section{REFERENCE}

1. Ziad, T., Nouri, H., Adny, A., Rochdi, Y., Aderdour, L., \& Raji, A. (2013). Miliaire tuberculeuse aiguë du pharynx ou maladie d'Isambert: à propos d'un cas. Archives de pédiatrie, 20(1), 41-43.

2. Gassab, E., Kedous, S., Berkaoui, A., Sayeh, N., Harrathi, K., Koubaa, J., \& Gassab, A. (2010). Tuberculose extra ganglionnaire de la tête et du cou. Journal Tunisien d'ORL et de Chirurgie Cervico-Faciale, 24(1).

3. Srirompotong, S., Yimtae, K., \& Jintakanon, D. (2004). Nasopharyngeal tuberculosis: manifestations between 1991 and 2000. Otolaryngology-Head and Neck Surgery, 131(5), 762-764.

4. Sawada, N., Inokuchi, G., Komatsu, H., Kurakawa, S., Tada, K., \& Kumoi, K. (2013). Nasopharyngeal tuberculosis. Journal of Infection and Chemotherapy, 19(6), 1158-1160.

5. Touati, M., Aljalil, A., Chihani, M., Bouchentouf, R., Bouaity, B., \& Ammar, H. (2013). La forme pseudo tumorale de la tuberculose primitive du nasopharynx: à propos de deux nouvelles observations et revue de la littérature. Pan African Medical Journal, 14(1).

6. Bouaity, B., Nadour, K., HEMMAOUI, B., ERRAMI, N., \& BENARIBA, F. (2009). Tuberculose du naso-pharynx: à propos d'un cas. La Lettre d'oto-rhino-laryngologie et de chirurgie cervico-faciale, (319), 14-16.

7. http://www.infovac-maroc.com/vaccins/1.BCG.pdf

8. Ouzdi, Z. A., Ahroui, Y., Zemrani, Y., Darouassi, Y., \& Ammar, H. (2019). Epidemiology of Otomycosis at the Military Hospital Avicenne of Marrakech (Morocco). Diabetes, 3, 10.

9. Bath, A. P., O'Flynn, P., \& Gibbin, K. P. (1992). Nasopharyngeal tuberculosis. The Journal of Laryngology \& Otology, 106(12), 1079-1080. 